Special issue of the 3rd International Conference on Computational and Experimental Science and Engineering (ICCESEN 2016)

\title{
Effect of Surfactant Types on the Size of Tin Oxide Nanoparticles
}

\author{
F. BORAN ${ }^{a}$, S. ÇETINKAYA ${ }^{b * *}$ AND M. ŞAHIN ${ }^{c}$ \\ ${ }^{a}$ Hitit University, Chemical Engineering Department, Çorum, Turkey \\ ${ }^{b}$ Cumhuriyet University, Chemical Engineering Department, Sivas, Turkey \\ ${ }^{c}$ Ankara University, Chemistry Department, Ankara, Turkey
}

\begin{abstract}
In this study, tin oxide $\left(\mathrm{SnO}_{2}\right)$ nanoparticles were synthesized by hydrothermal method in the presence of hydrazine and ammonia by adding surfactant for $12 \mathrm{~h}$ in a Teflon autoclave at $100^{\circ} \mathrm{C}$ reaction temperature. Tin(II) chloride hydrate as an inorganic precursor, hexadecyl trimethyl ammonium bromide (CTAB), and tetrapropyl ammonium bromide (TPAB) as cationic, and sodium dodecyl sulfonate (SDS) as anionic surfactants were used. The results showed that the size and shape of nanoparticles depended on the surfactant types. The nanoparticles sizes between 17.5 and $19.7 \mathrm{~nm}$ were obtained by changing types of surfactants. Synthesized tin oxide nanoparticles were characterized by field emission scanning electron microscopy, transmission electron microscopy, X-ray diffraction, and the Fourier transform infrared spectroscopy.
\end{abstract}

DOI: 10.12693/APhysPolA.132.546

PACS/topics: nanoparticle, tin oxide, surfactant

\section{Introduction}

The nanostructured materials have great significance in various areas of material engineering due to physical, mechanical, magnetic, and chemical properties [1]. The shape, small sizes and high surface-to-volume ratios are vital parameters for nanostructured materials. At the same time, the unique morphology and structure of semiconductor oxides can offer a lot of promising applications. Among them, as $n$-type of semiconductor, tin oxide has attracted great interest due to its properties such as wideband-gap $\left(E_{g}=3.6 \mathrm{eV}\right.$, at $\left.300 \mathrm{~K}\right)$, unique optical and electrical properties $[2,3] . \quad \mathrm{SnO}_{2}$ has great significance in widely technological applications such as dye-based solar cells, electrochromic devices [4], photovoltaics [3], transparent electrodes, catalysts, gas sensing, lithiumion batteries [2] and transistors [5]. There are several research methods to synthesize $\mathrm{SnO}_{2}$ nanostructures with high purity level, well dispersibility and controllable sizes, such as spray pyrolysis, hydrothermal process, evaporating tin grains in air, chemical vapor deposition, thermal evaporation of oxide powders, rapid oxidation of elemental tin and the sol-gel method, and hydrothermal method. Among these processes, hydrothermal method is a simple, energy economical and environmentally friendly process. The geometrical morphologies of nanoparticles that are synthesized with via hydrothermal process depend on the organic surfactant types. Also, the organic surfactants can be easily removed from the structure of the synthesized product by washing via alcohols or by calcination. Up to now, $\mathrm{SnO}_{2}$ nanostructures have been synthesized using surfactants with different charge (cationic,

*corresponding author; e-mail: cetinkaya.sevil@gmail.com anionic, non-ionic) and dosage, involved various geometrical morphologies such as nanorod, nanocube, nanowire, nanosheet, and nanoparticle. Therefore, it is very important to investigate the effect of surfactant types on the polar surfaces of $\mathrm{SnO}_{2}$ nuclei $[2,6]$. However, no previous research has been found that examines the effect of surfactant types on the size of $\mathrm{SnO}_{2}$ nanoparticles obtained by hydrazine-assisted hydrothermal process at low temperature.

This study attempts to provide some findings to this research area. Here, we aimed to obtain the smallest $\mathrm{SnO}_{2}$ nanoparticles with homogeneous distribution and different geometrical morphologies by hydrazineassisted hydrothermal process including cationic and anionic surfactants.

\section{Experimental}

$\mathrm{SnCl}_{2} \cdot 2 \mathrm{H}_{2} \mathrm{O}(10 \mathrm{mmol} \mathrm{g})$ was dissolved in $100 \mathrm{ml}$ deionized water at room temperature. $4 \mathrm{ml}$ ammonia solution (26\%) and $2.5 \mathrm{ml}$ hydrazine were added. After 20 min of stirring, the solution was subjected to ultrasonication for $1 \mathrm{~h}$. Then $0.5 \mathrm{mmol}$ CTAB, TPAB, and SDS were added to the solution. After ultrasonically dispersing the solution for $30 \mathrm{~min}$, hydrothermal treatment was carried out in a Teflon autoclave $(120 \mathrm{ml})$ at $100^{\circ} \mathrm{C}$ for $12 \mathrm{~h}$. Finally, the autoclave was cooled naturally to room temperature, the sample was filtered and washed with deionized water and pure ethanol until the effluent $\mathrm{pH}$ became 7 . The final product was dried at $60^{\circ} \mathrm{C}$ for $24 \mathrm{~h}$ and calcined at $500^{\circ} \mathrm{C}$ for $2 \mathrm{~h}$.

The morphologies of the tin oxide nanoparticles were observed by transmission electron microscopy (TEM, FEI Tecnai G2 Spirit Biotwin, 20-200 kV) and field emission scanning electron microscopy (FESEM, FEI Quanta 450 FEG, 20-30 kV). The crystal structure of the ob- 
tained nanoparticles was defined by X-ray power diffraction (XRD, Rigaku DMAX IIIC) with a $\mathrm{Cu} K_{\alpha} \mathrm{X}-$ ray source $(\lambda=1.541871 \AA)$ and the data were collected in the $2 \theta$ of $10-70^{\circ}$ at a scanning rate of $2^{\circ} / \mathrm{min}$. The Fourier transform infrared (FTIR)analysis was conducted using Mattson 1000 model FTIR spectrometer over a range from 400 to $4000 \mathrm{~cm}^{-1}$.

\section{Result and discussion}

Figure 1 shows the XRD patterns of the $\mathrm{SnO}_{2}$ nanoparticles by different surfactant assisted. All the diffraction peaks are attributed to the $\mathrm{SnO}_{2}$ rutile structure without observable impurity peaks (JCPDS card no. 41-1445) [6]. The three peaks at $2 \theta$ values of $26.6^{\circ}, 33.9^{\circ}$ and $51.9^{\circ}$ are associated with (110), (101) and (211) reflection planes of a tetragonal lattice of tin oxide [7]. The diffraction peaks at around $37.9^{\circ}, 54.7^{\circ}, 61.9^{\circ}$, and $65.9^{\circ}$ are associated with (200), (220), (310), and (301), respectively [6]. When XRD diagrams of $\mathrm{SnO}_{2}$ nanoparticles prepared using surfactant and no surfactant are compared, it is seen that the peak intensities are increasingly narrowing by adding surfactant. According to the results, $\mathrm{SnO}_{2}$ nanoparticles can be synthesized at different sizes using various surfactants.

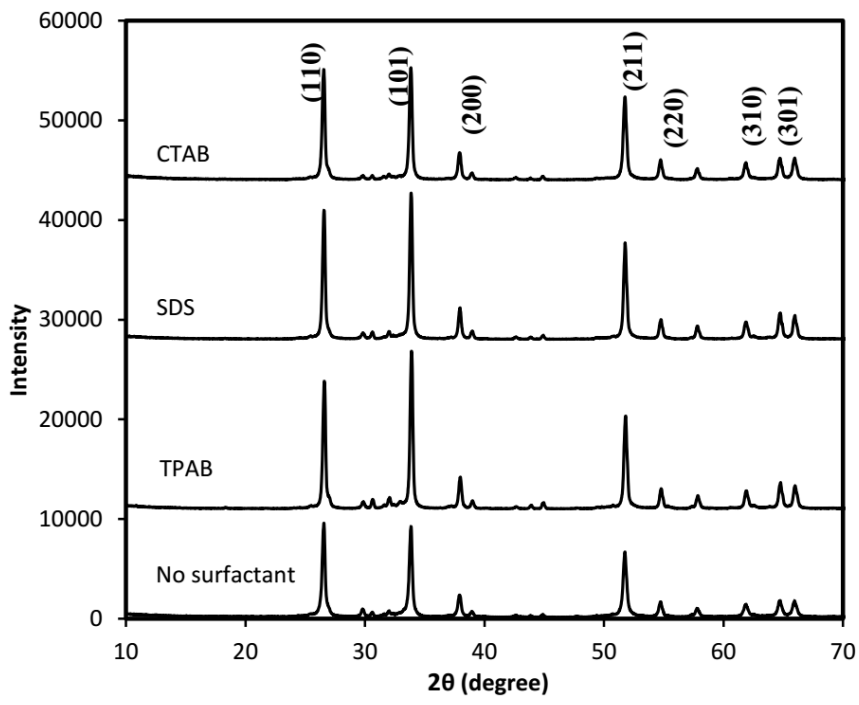

Fig. 1. XRD patterns of $\mathrm{SnO}_{2}$ nanoparticles by different surfactant assisted.

In Fig. 2, the FTIR spectrum of $\mathrm{SnO}_{2}$ nanoparticles by different surfactant assisted is illustrated. The band at $620 \mathrm{~cm}^{-1}$ can be attributed to a characteristic peak of the oxide-bridge functional group $(\mathrm{O}-\mathrm{Sn}-\mathrm{O})$. In addition, the peaks around $3430 \mathrm{~cm}^{-1}$ appeared due to stretching vibration of adsorbed water at the surface of tin oxide [7].

The effect of surfactant types on the surface morphology and size of $\mathrm{SnO}_{2}$ nanoparticles was observed by FESEM (Fig. 3) and TEM (Fig. 4). As shown in Fig. 3, densely packed and irregularly spherical-like $\mathrm{SnO}_{2}$ nanoparticles were noticed. But, the exact shape of each

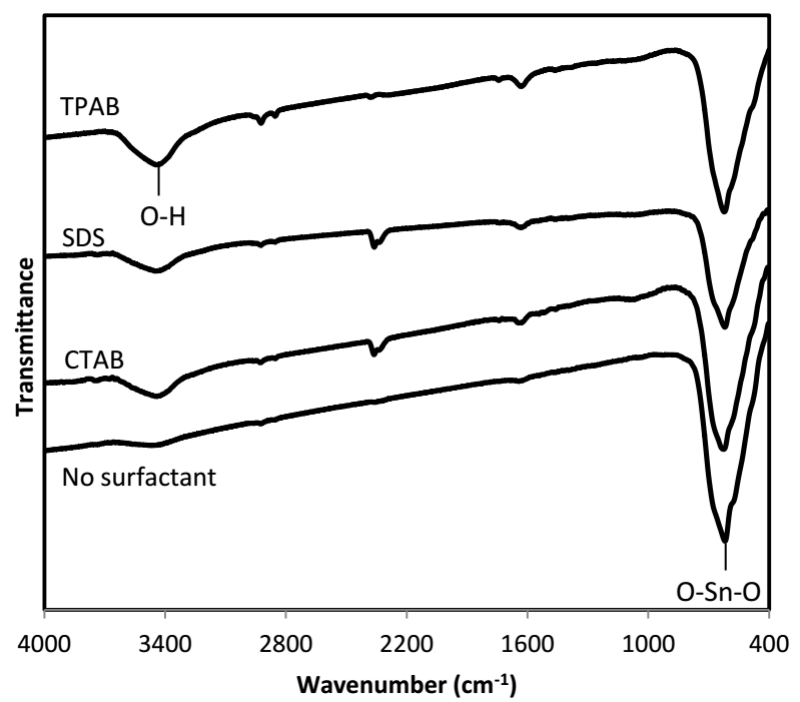

Fig. 2. FTIR spectra of $\mathrm{SnO}_{2}$ nanoparticles by different surfactant assisted.

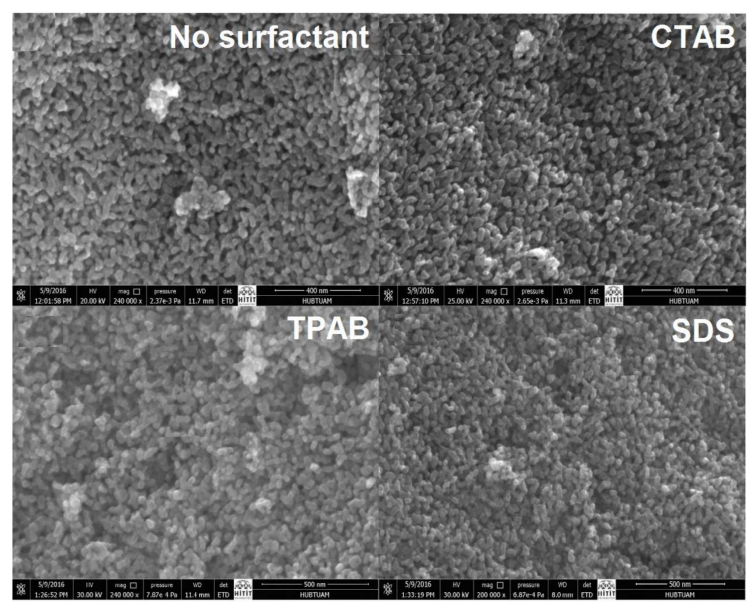

Fig. 3. FESEM micrograph of $\mathrm{SnO}_{2}$ nanoparticles by different surfactant assisted.

particle cannot be understood from the FESEM photos. In addition, as seen in Fig. 4, the $\mathrm{SnO}_{2}$ nanoparticles synthesized without surfactant were stacked together and composed mainly of nanospheres structures. The shape of $\mathrm{SnO}_{2}$ nanoparticles were also influenced by varying surfactant type such as nanorectangles and nanohexagons when we used SDS, CTAB, and TPAB, respectively. Shapes of the particles were formed in nanohexagonal structures by the addition of CTAB and TPAB cationic surfactants to the synthesis medium. But, it was observed that the effect of adding the surfactants formed much $\mathrm{SnO}_{2}$ nanoparticles stacked together. By adding SDS anionic surfactant to the synthesis medium, nanorectangles were obtained and the particle accumulation could be avoided at some point. As a result of that, particles in shape of nanorectangles were obtained. According to TEM results, the sizes of $\mathrm{SnO}_{2}$ nanoparticles were determined using the UTHSCSA Image Tool 3 image analysis program [8]. The average 
size of $\mathrm{SnO}_{2}$ nanoparticles was obtained as 19.7, 18.2, 19.2 , and $17.5 \mathrm{~nm}$ by changing surfactant types as no surfactant, CTAB, SDS, and TPAB, respectively. The smallest particle size and the best particle distribution were achieved by using TPAB $(17.5 \mathrm{~nm})$ as cationic surfactant and SDS as anionic surfactant, respectively (Fig. 4). These results are due to the combined interplay between the electrostatic interaction and the Van der Waals' forces [2].

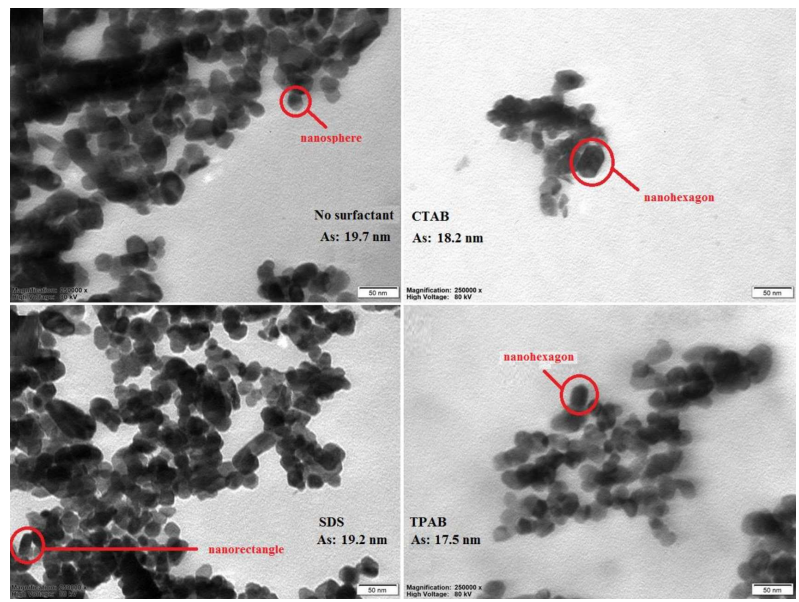

Fig. 4. TEM images of $\mathrm{SnO}_{2}$ nanoparticles by different surfactant assisted.

\section{Conclusion}

We have successfully prepared $\mathrm{SnO}_{2}$ nanoparticles with homogeneous distribution and different geometrical morphologies by hydrazine-assisted hydrothermal process including cationic (CTAB, TPAB) and anionic (SDS) surfactants. The effects of surfactant types on the surface morphology and size of $\mathrm{SnO}_{2}$ nanoparticles were investigated. The average particle size was obtained in the range of 17.5 to $19.7 \mathrm{~nm}$. While $\mathrm{SnO}_{2}$ nanospheres was prepared without surfactant, the shape of $\mathrm{SnO}_{2}$ nanoparticles was achieved as nanorectangles and nanohexagons using anionic and cationic surfactants, respectively. TPAB and SDS provided the smallest particle size and the better particle distribution of $\mathrm{SnO}_{2}$, respectively.

\section{Acknowledgments}

This work was supported by Cumhuriyet University Scientific Research Project (CUBAP), project numbered as M601.

\section{References}

[1] W. Laslounia, Z. Hamlatia, M. Azzaz, Acta Phys. Pol. A 128, B-190 (2015).

[2] M. Wang, Y. Gao, L. Dai, C. Cao, X. Guo, J. Solid State Chem. 189, 49 (2012).

[3] P. Sun, X. Mei, Y. Cai, J. Ma, Y. Sun, X. Liang, F. Liu, G. Lu, Sens. Actuat. B 187, 301 (2013).

[4] S.P. Lim, N.M. Huang, H.N. Lim, Ceram. Int. 39, 6647 (2013).

[5] P. Sun, Y. Cao, J. Liu, Y. Sun, J. Ma, G. Lu, Sens. Actuat. B 156, 779 (2011).

[6] G.E. Patil, D.D. Kajale, V.B. Gaikwad, G.H. Jain, Int. Nano Lett. 2, 17 (2012).

[7] M.A. Farrukh, B.-T. Heng, R. Adnan, Turk. J. Chem. 34, 537 (2010).

[8] A. Alemdar, M. Sain, Biores. Technol. 99, 1664 (2008). 